\title{
A Sodium Selective Electrochemical Biosensor Based on the Sodium Pump
}

\author{
Li Longjie, Zhao Hongshen, Ji Liangliang, Ma Baofu, Chen Deliang* \\ College of Life Sciences, University of Chinese Academy of Sciences, Beijing, China
}

\section{Email address:}

lilongjie13@mails.ucas.ac.cn (Li Longjie), zhaohongshen12@mails.ucas.ac.cn (Zhao Hongshen),

jiliangliang14@mails.ucas.ac.cn (Ji Liangliang), mabaofu15@mails.ucas.ac.cn (Ma Baofu), dlchen@ucas.ac.cn (Chen Deliang)

${ }^{*}$ Corresponding author

\section{To cite this article:}

Li Longjie, Zhao Hongshen, Ji Liangliang, Ma Baofu, Chen Deliang. A Sodium Selective Electrochemical Biosensor Based on the Sodium Pump. Science Discovery. Vol. 4, No. 4, 2016, pp. 238-242. doi: 10.11648/j.sd.20160404.16

Received: June 6, 2016; Accepted: July 5, 2016; Published: July 20, 2016

\begin{abstract}
Na}^{+}$is one of the most abundant metal ions and plays critical physiological roles in biological systems, therefore development of new $\mathrm{Na}^{+}$sensors is becoming increasingly important. Currently many physical and chemical methods have been applied to detect $\mathrm{Na}^{+}$. In this paper, we developed a novel $\mathrm{Na}^{+}$electrode based on a biomaterial, light-driven $\mathrm{Na}^{+}$pump $(\mathrm{NdR} 2)$. The photocurrent of the electrode upon excitation shows a characteristic positive polarity in $\mathrm{Na}^{+}$solution at neutral $\mathrm{pH}$. The peak current shows $\mathrm{Na}^{+}$dependency within the concentration range from 1 to $200 \mathrm{mmol} / \mathrm{L}$. The stability data show that the electrode is stable after being stored at $50^{\circ} \mathrm{C}$ for $90 \mathrm{~min}$. As a novel biosensor, this $\mathrm{Na}^{+}$pump-based electrode is of great significance in the study of ion transport mechanism and application of ion detection.
\end{abstract}

Keywords: NdR2, NdR2-liposome-ITO Electrode, $\mathrm{Na}^{+}$Concentration, Biosensor

\section{一种基于 $\mathrm{Na}^{+}$泵的钠离子选择性电化学生物传感器}

\author{
李龙杰, 赵宏深, 季亮亮, 马宝福, 陈德亮* \\ 生命科学学院, 中国科学院大学, 北京, 中国 \\ 邮箱 \\ lilongjie13@mails. ucas. ac. cn（李龙杰），zhaohongshen12@mails.ucas. ac. cn（赵宏深）, \\ jiliangliang14@mails. ucas. ac. cn（季亮亮）, mabaofu15@mails. ucas. ac. cn（马宝福），dlchen@ucas. ac. cn（陈德亮）
}

摘要: 钠离子作为人体生理代谢中非常重要的离子, 对其进行检测非常重要。目前国际上检测钠离子的方法有许多, 但主要是利用物理、化学方法。本文将一种光驱动的 $\mathrm{Na}^{+}$转运蛋白 (NdR2) 与氧化铟锡导电玻璃（IT0）结合, 制备成 生物电极, 对 $\mathrm{Na}^{+}$进行检测。结果表明, 此电极对 $\mathrm{Na}^{+}$有特征性的响应, 电流峰值呈现 $\mathrm{Na}^{+}$浓度依赖性（1 200mM）。稳定 性试验表明, $50^{\circ} \mathrm{C}$ 处理 $90 \mathrm{~min}$, 不影响电极探测 $\mathrm{Na}{ }^{+}$浓度的功能。作为全新的钠离子探测生物传感器、 $\mathrm{Na}^{+}$转运蛋白电极, 在理论和应用领域都具备重要的研究意义。

关键词: $\mathrm{NdR} 2, \mathrm{NdR} 2-$ 脂质体-IT0电极, $\mathrm{Na}^{+}$浓度, 生物传感器 


\section{1. 前言}

$\mathrm{Na}^{+}$作为生命体中极为重要的离子, 参与神经传导、 肌肉运动、新陈代谢等生理活动。因此, $\mathrm{Na}^{+}$的定量检测 在基础理论及应用领域都具有非常重要的研究意义。目前 国际上检测 $\mathrm{Na}^{*}$ 的方法有许多, 包括离子选择性电极、原 子吸收光谱法 $[1]$ 、苂光显微镜 $[2] 、{ }^{23} \mathrm{Na}$ 核磁共振 $[3]$ 等一 系列物理、化学方法。2015年PNAS上首次报道了利用生物 学原理探测 $\mathrm{Na}^{+}$浓度的方法——Torabi等人 [4] 发现一种 钠离子特异性DNAzyme, 其结合 $\mathrm{Na}^{+}$之后能够对DNA进行剪 切, 从而提出了一种基于生物材料的检测 $\mathrm{Na}^{+}$浓度的方法。

本文的工作则首次提出将视紫红质家族中的一种全 新蛋白一一钠离子泵NdR2, 与电化学的方法相结合, 设计 了一种基于蛋白质的稳定的钠离子选择性电化学生物传 感器。

视紫红质是一种结合视黄醛配基的膜蛋白, 以七次 $a-$ 螺旋结构插入细胞膜内。视黄醛位于七次跨膜螺旋结 构的中心, 通过希夫碱基与第七螺旋上的赖氨酸残基形成 共价连接 $[5]$ 。

视紫红质在光作用下能产生电信号, 这一现象在早期 研究细菌视紫红质BR光驱动质子转运特性时就已发现。视 紫红质蛋白在传递离子时, 内部构象的变化所导致的电荷 分布变化以及离子在内部的定向传递, 都会使蛋白质两侧 产生电势变化 $[6,7]$ 。将视紫红质固定化处理于电极表面, 可用于探测光驱动的电流信号。

基于这种光电响应的性质，视紫红质家族在信息探测、 生物传感、太阳能电池 $[8]$ 、人工视网膜 $[9,10]$ 等方面具 有众多的实际应用与广阔的研究前景。Choi等 [11]研究了 由 $B R /$ 黄素组成的电化学光电池, 认为其可作为颜色识别 的人工光感受器, 能模仿人类视觉系统的信息处理。

Ahmadi等 [12]设计制造出一种基于BR的传感器来检 测 $X$-射线辐射, 通过辐射诱导的电流来监测 $X$-射线辐射。 Rao等 $[13,14]$ 利用变形菌视紫红质 $\mathrm{PR}$ 的 $\mathrm{pH}$ 依赖的光电特 性, 设计出了一种 $\mathrm{pH}$ 电化学生物传感器, 与其他基于荧光、 磷光、比色的方法比较, 这种传感器能够快速、高敏感地 实时探测溶液 $\mathrm{pH}$ 的变化。

NdR2 是2013年在海洋黄杆菌Nonlabens dokdonensis 的细胞膜上发现新的视紫红质 [15], 其功能目前没有报道, 而本实验室的前期工作显示, NdR2 是一个光驱动的钠离子 原。基于该家族蛋白的光电响应特性, 本文通过将NdR2脂质体与氧化铟锡导电玻璃结合制备成NdR2-脂质体-IT0 电极, 实验发现: 该电极对 $\mathrm{Na}^{+} 、 \mathrm{Li}^{+}$有特异性的正电流响 应, 并具有浓度依赖性（1mM 200mM）; 电极经过较高温 度 $\left(50^{\circ} \mathrm{C}\right)$ 处理 $90 \mathrm{~min}$ 后, 仍然对 $\mathrm{Na}^{+}$保持特异性响应并能 检测 $\mathrm{Na}^{+}$浓度。

\section{2. 实验方法}

\section{1. NdR2 的提取纯化}

培养大肠杆菌表达NdR2, 离心, 收集细菌。用Lysis buffer (50mM Tris-HCl, 1mM EDTA-Na, $100 \mathrm{mM} \mathrm{KCl,} 1 \mathrm{mM}$ DTT, pH8.0）重悬细菌, 离心。用Lysis buffer充分悬浮
细菌, 加入Lysozyme ( $5 \mathrm{mg} / 30 \mathrm{ml})$ 和 PMSF, 裂解细菌。 再用超声继续破碎细菌, 离心收集上清液（红色）。将上 清液高速离心收集沉淀 (红色)。用Solubilizing buffer (10mM Tris-HCl, 400mM KCl, pH8.0) 洗涤1次, 离心后 再用含DDM的Solubilizing buffer (10mM Tris- $\mathrm{HCl}, 400 \mathrm{mM}$ $\mathrm{KCl}, 1 \%$ 的DDM, pH8.0) 溶解, $4^{\circ} \mathrm{C}$ 避光匀浆。高速离心, 收集上清液（红色），加入imidazole（pH8.0），其终浓 度为 $20 \mathrm{mM}$ 。用大量去离子水洗涤 $\mathrm{Ni}-\mathrm{NTA}$, 再用 10 倍柱体积 Equilibrium buffer (10mM Tris- $\mathrm{HCl} ， 400 \mathrm{mM} \mathrm{KCl，0.05 \%}$ DDM, 10mM imidazole, pH8.0) 洗涤。将上述红色上清加 入Ni-NTA中纯化。用20倍柱体积的Washing buffer (10mM Tris-HCl, 400mM KCl, 0. 05\%DDM, 20mM imidazole, pH8. 0) 洗涤。用Elution buffer (10mM Tris-HCl, 400mM KCl, 0.05\% DDM, 500mM imidazole, pH8.0) 洗脱, 收集洗脱 液, $4^{\circ} \mathrm{C}$ 浓缩、透析。透析液 ( $5 \mathrm{mM}$ Tris $-\mathrm{HCl}, 200 \mathrm{mM} \mathrm{KCl}$, pH8.0）。光谱测定浓缩透析后的蛋白浓度。浓度计算方 法: 根据 $A=E \cdot 1 \cdot c, A$ 为特征吸收峰处的吸光值, 一般 $1=1 \mathrm{~cm}$, 对于 $N d R 2 （ 520 \sim 530 \mathrm{~nm} ）, \varepsilon=50000$ 。

\section{2. NdR2-脂质体的制备}

称取大豆卵磷脂、胆固醇、维生素 $\mathrm{E}$ (质量比550:50:1), 加入乙醚充分溶解。旋转蒸发, 真空原抽气, 得到薄层膜 状磷脂贴于玻璃瓶内壁。用氮气吹薄膜表面, 使乙醚完全 挥发。加入buffer (0.1 M Tris-Tris-Cl, pH 8.0), 使 用超声仪超声, 使磷脂清澈, $\mathrm{OD}_{550}=0.45$ 。向磷脂溶液中 加入Triton X-100, 混匀 (最终Triton X-100的浓度为 $0.5 \%$ ）。将NdR2蛋白（ $5 \mathrm{mM} \mathrm{Tris}-\mathrm{Cl}, 200 \mathrm{mM} \mathrm{KCl}, 0.05 \%$ DDM, pH 8.0) 与脂质体溶液混合, 室温用混匀器震荡 $60 \mathrm{~min}$ 。 其中, 蛋白: 磷脂=8: 10 (质量比), 即摩尔比为 $1: 50$ 。 $\mathrm{NdR} 2$ 终浓度为 $2 \mathrm{mg} / \mathrm{ml}$ 。60min后加入bio-beads 去除去垢剂。 用水稀释样品, 离心, 用水重悬。 $\mathrm{NdR} 2$ 终浓度为 $4 \mathrm{mg} / \mathrm{m} 1$ 。

\section{3. 光电流测定方法}

将IT0导电玻璃裁剪成小块, 先后用丙酮、乙醇、水 进行超声清洗。清洗完毕后吹干, 坚立保存待用。裁剪合 适大小的硅胶, 用打孔器打出圆孔, 将硅胶吸附于ITO的 导电侧。吸取样品滴加到圆孔中, 避免出现气泡。置于暗 处, 待样品的水分蒸发完全电极即制备完成。调试好光电 流检测系统, 将制备完成的电极与一个不含蛋白样品的对 电极分别放到三层聚四氟乙烯板的中间, 通过中间的聚四 氟乙烯板上的小孔将电解液 ( $\mathrm{LiCl} 、 \mathrm{NaCl} 、 \mathrm{KCl} 、 \mathrm{CsCl}$ 、 $\mathrm{CaCl}_{2} 、 \mathrm{MgCl}_{2} 、 \mathrm{NaF} 、 \mathrm{Na}_{2} \mathrm{SO}_{4} 、 \mathrm{~K}_{2} \mathrm{SO}_{4}$ ) 注射到两个电极之间, 放置于光电流检测系统中检测。

\section{3. 实验结果与讨论}

\section{1. NdR2, NdR2-脂质体及NdR2-脂质体-IT0电极的表征}

中性的NdR2-DDM溶液为红色 (1A), 而制备成的电极, 由于空气中 $\mathrm{CO}_{2}$ 存在等原因, 导致电极表面的 NdR2-脂质体 呈酸性, 颜色为蓝紫色 (1B)。将电极置于中性溶液中平 衡一段时间后, 颜色恢复 (1C)。 


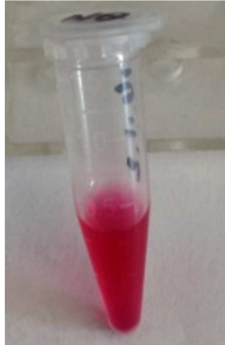

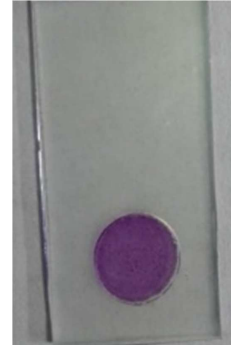

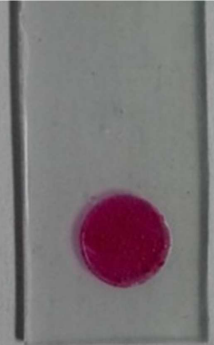

图1 NdR2和NdR2-脂质体-ITO电极的实物图（A，B，C）。

采用负染色技术对NdR2进行染色, 用透射电子显微镜 (TEM) 对蛋白进行观察发现，表面活性剂DDM包裹的NdR2 尺寸大概在 $20 \mathrm{~nm}$ 左右，如图2A所示。

表面活性剂包裹的蛋白质相比于脂质重组状态, 在溶 液中更加稳定，不容易形成蛋白聚集或沉降。但表面活性 剂渗透性较强, 无法有效地维持离子浓度梯度。因此, 为 了有效利用NdR2 的光驱动离子泵特性, 需要将其组装到更 利于离子浓度梯度形成的微环境中。另外, 脂质重组蛋白 与材料的结合力会增强, 不易从ITO上脱落。因此, 本实 验选择了脂质体对NdR2进行重组。图2B显示, 空脂质体的 大小约为几十至几百纳米。

氧化铟锡导电玻璃（ITO）有透明、易裁剪、导电侧 电阻小等优点, 将制备好的NdR2-脂质体自然沉积到 ITO 上, 作为工作电极, 置于光电响应检测系统中进行光电流 的检测。通过扫描电镜 (SEM) 可以观察到, 沉积NdR2脂质体后 ITO表面具有尺寸几十至几百纳米大小的球状颗 粒 (Data no shown)。

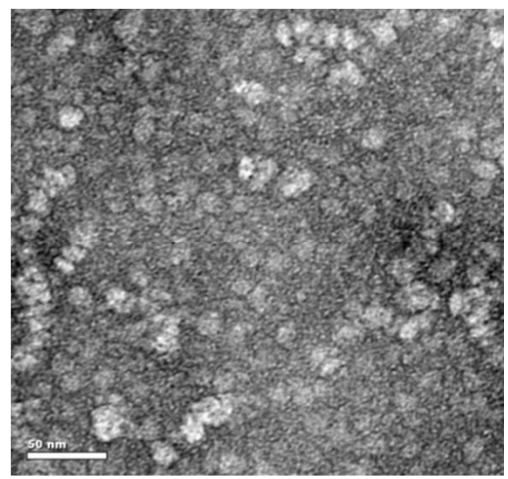

A

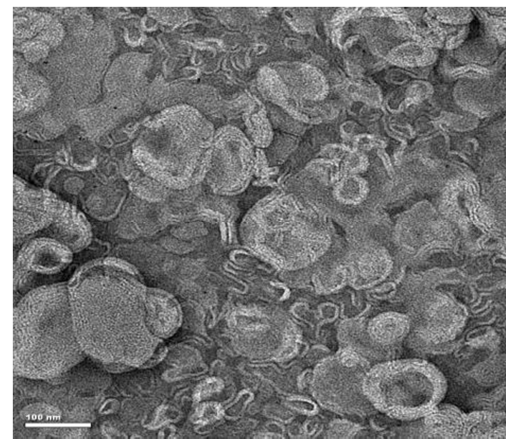

B

图2 透射电子显微镜（TEM）下的NdR2-DDM（A）及空脂质体（B）。

\section{2. NdR2-脂质体-ITO电极的离子选择性与 $\mathrm{Na}^{+}$浓度依赖 性}

Inoue等人 $[16,17]$ 曾报道一种NdR2 的同源蛋白KR2， 光照下在不同的阳离子溶液中行驶不同的功能, 在 $\mathrm{Li}^{+}$或 $\mathrm{Na}^{+}$溶液中, 它将 $\mathrm{Li}^{+}$或 $\mathrm{Na}^{+}$从蛋白C端向 $\mathrm{N}$ 端传递, 而在其他 阳离子 $\left(\mathrm{K}^{+} 、 \mathrm{Rb}^{+} 、 \mathrm{Cs}^{+}\right)$溶液中, 只进行 $\mathrm{H}^{+}$的传递。

本实验室前期的工作也证实, NdR2 也具有与KR2相似 的功能——在 $\mathrm{Li}^{+}$或 $\mathrm{Na}^{+}$溶液中为 $\mathrm{Li}^{+}$泵或 $\mathrm{Na}^{+}$百, 而在其他溶 液中为 $\mathrm{H}^{+}$洜。

将NdR2-脂质体-IT0电极置于光电响应检测系统中, 检测光电流信号。实验结果显示, 当阳离子为 $\mathrm{Na}^{+}(100 \mathrm{mM})$ 时, 光电流信号的极性为正, 如图3所示, 且无论阴离子 $\left(\mathrm{Cl}^{-} 、 \mathrm{~F}^{-} 、 \mathrm{SO}_{4}{ }^{2-}\right)$ 为何, 保持 $\mathrm{Na}^{+}$浓度相同时其电信号幅度 基本一致; 而光电流信号的极性在阳离子为 $\mathrm{K}^{+} 、 \mathrm{Ca}^{2+} 、 \mathrm{Mg}^{2+}$ 、 $\mathrm{Cs}^{+}$时相同, 均为负信号 (Some data no shown)。因此 我们认为, NdR2-脂质体-IT0电极对 $\mathrm{Na}^{+}$有特异性的响应, 这与本实验室之前利用 $\mathrm{pH}$ 测定证实 $\mathrm{NdR} 2$ 具有 $\mathrm{Na}^{+}$转运功能 的观点一致。

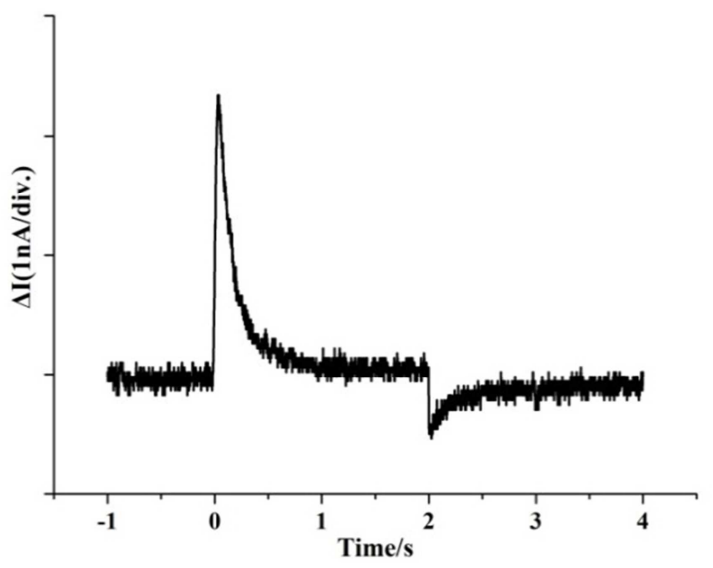

图3 NdR2-脂质体-IT0电极对 $\mathrm{Na}^{+}$溶液 (100mM) 的特征性响应。0s时光照, $2 \mathrm{~s}$ 时避光。

为了定量地评估NdR2-脂质体-IT0电极对 $\mathrm{Na}^{+}$的响应, 我们检测其在不同浓度的 $\mathrm{Na}^{+}$溶液 (1 200mM) 中的光电流。 由于每一个电极的光电流都不尽相同, 所以在进行统计时, 需要将光电流标准化, 即以每个电极探测的光电流值除以 本组最大光电流值, 得到标准电流值。如图4所示, 随着 溶液中 $\mathrm{Na}^{+}$浓度的增加, 光电流具有显著的下降趋势。

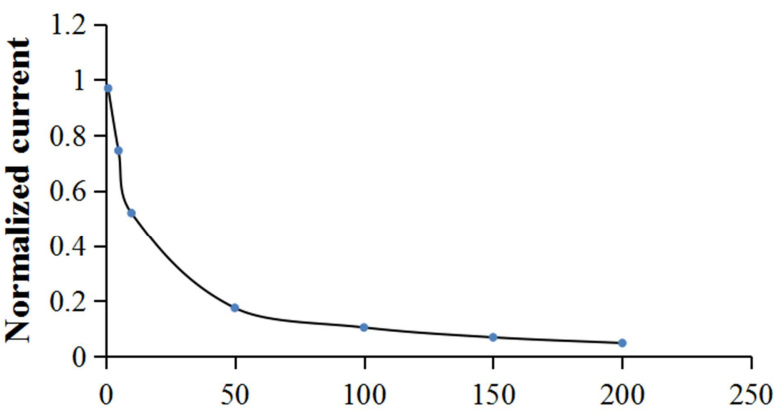

$\mathrm{Na}^{+}$concentration $/ \mathbf{m m o l} \cdot \mathbf{L}^{-1}$

图4 NdR2-脂质体-IT0电极对不同浓度Na*溶液（pH7.5）的响应。 


\section{3. NdR2-脂质体-IT0电极的热稳定性}

作为一个生物传感器, 其稳定性受到广泛关注, 因此 本文对NdR2-脂质体-IT0电极的热稳定性作了一定的评估。

在热稳定性的实验中, 将电极置于 $50^{\circ} \mathrm{C}$ 的水浴中 90 分钟, 水浴前后, 光电流的变化很小, 经过热处理之后的 电极对 $\mathrm{Na}^{+}$浓度具有依赖性, 如图5所示。不仅如此, 即使 经过 $70^{\circ} \mathrm{C}$ 的高温处理 90 分钟, 电极仍然对 $\mathrm{Na}^{+}$浓度具有依 赖性（Data no shown）。

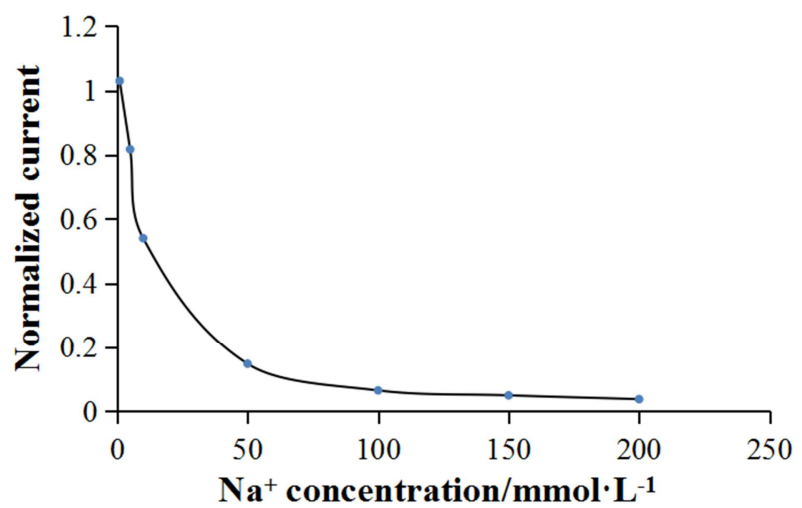

图5 50 ${ }^{\circ} \mathrm{C}$ 处理 $90 \mathrm{~min}$ 的 $\mathrm{NdR} 2-$ 脂质体-IT0电极对不同浓度 $\mathrm{Na}^{*}$ 溶液（pH7.5） 的响应。

\section{4. NdR2-脂质体-ITO电极对 $\mathrm{Na}^{+}$溶液}

利用经标准液标定过的电极来检测 $\mathrm{Na}^{+}$溶液样品, 通 过对比检测值与实际值来分析电极的测定精确度。将检测 得到的光电流值代入标准曲线, 计算出这些试验溶液中的 $\mathrm{Na}^{+}$浓度值, 如表1所示。从表中可以看出, 样品的 $\mathrm{Na}^{+}{ }^{+}$农度 检测值接近于实际值，其回收率为 $93 \%$ ，表明已建立的生 物传感电极能够检测 $\mathrm{Na}^{+}$的实际浓度。此外, 我们也检测 了热处理后电极的测定精确度, 回收率为从 $91 \%$, 这也进 一步证明此电极具有良好的稳定性。

表 $1 \mathrm{Na}^{+}$溶液样品的实际值与检测值。样品包含 $1 \mathrm{mmol} / \mathrm{L}$ Tris-HC1, pH7. 5 。

\begin{tabular}{lllll}
\hline \multirow{2}{*}{$\begin{array}{l}\text { Actual } \\
\text { value } / \mathrm{mmol} \cdot \mathrm{L}^{-1}\end{array}$} & \multicolumn{2}{l}{ Test value/mmol $\bullet \mathrm{L}^{-1}$} & \multicolumn{2}{l}{ Correlation(\%) } \\
\cline { 2 - 5 } & $\mathrm{RT}$ & $\mathbf{5 0} \mathrm{C} \mathbf{9 0 m i n}$ & $\mathrm{RT}$ & $\mathbf{5 0}{ }^{\circ} \mathrm{C}$ 90min \\
\hline \multirow{2}{*}{40} & 37.16 & 36.24 & 93 & 91 \\
\hline
\end{tabular}

RT: room temperature, 室温。

\section{4. 结束语}

生物传感器是以具有生物活性的物质 (DNA、蛋白质、 酶等) 作探测元件与一些物理或化学元件结合而组成的一 种检测装置, 它在临床医学诊断、环境监测、食品检测等 方面都具有广泛的应用。

本文通过将一种新型的视紫红质- - NdR2 与氧化铟 锡导电玻璃结合制备成生物电极, 通过一系列实验得到以 下结论:

（1） NdR2-脂质体-IT0电极对 $\mathrm{Na}^{+}$有特异性的正电流 响应。
（2）随着 $\mathrm{Na}^{+}$浓度的上升, $\mathrm{NdR2}-$ 脂质体-IT0电极的 光电流信号不断减小, 并且在一定范围内 ( $1 \mathrm{mM} 200 \mathrm{mM})$, $\mathrm{Na}^{\star}$ 浓度与光电流呈一定的函数关系。

(3) $\mathrm{NdR2}$-脂质体-IT0电极经过较高温度 $\left(50^{\circ} \mathrm{C}\right)$ 处 理90min后, 仍然对于 $\mathrm{Na}^{+}$保持特异性响应并能检测 $\mathrm{Na}^{+}$浓度。

本文尝试着对钠离子选择性电化学生物传感器进行了 初步性的探索, 工作仍有许多不完善的地方, 有许多问题需 要回答与解决, 例如, 如何提高对生物样品中钠离子检测的 精确度, 如何提升电极对钠离子浓度更大范围的响应, 等等。 期待利用更多的物理、化学手段和材料, 与NNdR2-脂质体-IT0 电极相结合, 创造出更加完善的电化学生物传感器。

\section{致谢}

本文为国家自然科学基金面上项目 (Y21101Q1A1, Y31101M1A1) 的阶段性成果之一。

\section{参考文献}

[1] Malloy, C. R. et al. Influence of Global-Ischemia on Intracellular Sodium in the Perfused Rat-Heart [J]. Magnet Reson Med, 1990, 15: 33-44.

[2] Grubman, A. et al. X-ray fluorescence imaging reveals subcellular biometal disturbances in a childhood neurodegenerative disorder $[J]$. Chem Sci, 2014, 5: 2503-2516.

[3] Ivanics, T., Blum, H., Wroblewski, K., Wang, D. J. \& 0sbakken, M. Intracellular Sodium in Cardiomyocytes Using Na-23 Nuclear-Magnetic-Resonance [J]. Bba-Mol Cel1 Res, 1994, 1221: 133-144.

[4] Torabi, S. F. et al. In vitro selection of a sodium-specific DNAzyme and its application in intracellular sensing $[\mathrm{J}]$. Proceedings of the National Academy of Sciences of the United States of America, 2015, 112: 5903-5908.

[5] Oesterhelt, D. \& Stoeckenius, W. Rhodopsin-like protein from the purple membrane of Halobacterium halobium [J]. Nature: New biology, 1971, 233: 149-152.

[6] Liu, S. Y. \& Ebrey, T. G. Photocurrent measurements of the purple membrane oriented in a polyacrylamide gel [J]. Biophys J, 1988, 54: 321-329.

[7] Cao, Y. et al. Water is required for proton transfer from aspartate-96 to the bacteriorhodopsin Schiff base [J]. Biochemistry, 1991, 30: 10972-10979.

[8] Bertoncello, P., Nicolini, D., Paternolli, C., Bavastrello, V. \& Nicolini, C. Bacteriorhodopsin-based Langmuir-Schaefer films for solar energy capture [J]. IEEE transactions on nanobioscience, 2003, 2: 124-132. 
[9] Hong, F. T. Molecular Sensors based on the photovoltaic effect of bacteriorhodopsin: origin of differential responsivity $[\mathrm{J}]$. Materials Science and Engineering, 1997.

[10] Miyasaka, T., Koyama, K. \& Itoh, I. Quantum conversion and image detection by a bacteriorhodopsin-based artificial photoreceptor [J]. Science, 1992, 255: 342-344.

[11] Choi, H. G., Jung, W. C., Min, J. H., Lee, W. H. \& Choi, J. W. Color image detection by biomolecular photoreceptor using bacteriorhodopsin-based complex LB films [J]. Biosensors \& bioelectronics, 2001, 16: 925-935.

[12] Ahmadi, M. \& Yeow, J. T. Fabrication and characterization of a radiation sensor based on bacteriorhodopsin [J]. Biosensors \& bioelectronics, 2001, 26: 2171-2176.
[13] Rao, S. et al. A proteorhodopsin-based biohybrid light-powering $\mathrm{pH}$ sensor $[\mathrm{J}]$. Physical chemistry chemical physics, 2013, 15: 15821-15824.

[14] Rao, S. et al. A light-powered bio-capacitor with nanochannel modulation $[\mathrm{J}]$. Advanced materials, 2014, 26: $5846-5850$.

[15] Kwon, S. K. et al. Genomic makeup of the marine flavobacterium Nonlabens (Donghaeana) dokdonensis and identification of a novel class of rhodopsins [J]. Genome biology and evolution, 2013, 5: 187-199.

[16] Inoue, K. et al. A light-driven sodium ion pump in marine bacteria $[\mathrm{J}]$. Nature communications, 2013, 4: 1678.

[17] Kato, H. E. et al. Structural basis for Na transport mechanism by a light-driven Na pump [J]. Nature, 2015. 